\title{
Students' Views of Math and Physics Problems: Structure vs. Content
}

\author{
Dyan L. Jones* \\ *Department of Physics, Mercyhurst University, 501 E. $38^{\text {th }}$ Street, Erie, PA 16546
}

\begin{abstract}
This is a continuation of a set of work investigating how students use mathematics when solving physics problems. This phase of the study specifically looked at qualitative data from in-depth group interviews to expand upon the more quantitative studies previously conducted. Specifically, but unsurprisingly, the results indicate that students tend to focus on the solution technique when examining math problems but heavily emphasize the context of the problem when examining physics problems. This is evident in a variety of tasks including grouping/matching exercises and oral discussions of the nature of the problems, and is true regardless of the purposeful prompting of students to consider the solution techniques required. Analysis from these interviews will be presented here, as well as some indication for future work to facilitate a more natural and useful incorporation of these disciplines for effective problem solving.
\end{abstract}

Keywords: physics education, math, structure, content, similarity, problem solving

PACS: 01.40.Fk

\section{INTRODUCTION}

As has been described many times, there is a clear need for students to be able to use the 'tools' of mathematics in order to successfully solve physics problems at the university level. The difficulties that students have with mathematics in the calculus-based introductory sequence have been documented in a variety of places, including numerous papers in these proceedings over the past few years. Still, many questions remain unasked as we move toward a more complete body of research and design possible interventions to assist student learning in these areas.

The work presented here is a continuation of an ongoing project to further these studies by examining how students view the nature of math and physics problems and in what ways they use the tools provided by mathematics to help them solve physics problems.

\section{LITERATURE REVIEW}

Problem solving is a heavy phrase - it takes on a plethora of different meanings and connotations, and the study of problem solving in its various forms pervades the literature, to the point that a full discussion here is impossible. However, by narrowing our attention to those particularly focused on the interaction of mathematics, we see an increasing trend.

Bassok and Holyoak provided one of the initial studies in 1989, in which they studied the effects of presenting either the mathematics or physics first. ${ }^{1}$ Other work has shown that despite recognizing similar problems, students do not necessarily show improved problem-solving performance. $^{2}$ Redish and colleagues have studied the different representations used between disciplines as one potential factor in student difficulties. ${ }^{3}$ When it comes to the specifics of mathematics, there is a clear leaning toward the meaning and implication of integrals. ${ }^{4,5}$

One of the outcomes of the work presented here is that students seem to focus on structure and context differently in the two types of problems. The literature in this realm is also broad and varied; provided here is a cursory review. Work by Chi, Feltovich and Glaser was perhaps the first major study in this area. ${ }^{6}$ By asking both experts and novices to identify similarities between problems, they found that novices tended to focus on the surface features of a problem while experts focused on the physics principles being used. These studies were later supported by a number of works, though one notable study indicated that even experts may at times be misled by the problem structure. ${ }^{7}$ More recent work by Singh also shows that one notable difference between experts and novices is that experts are able to propose a variety of problem solving options for a single task, while novices rely on only one solution technique. ${ }^{8}$ Finally, Hegarty et al. looked specifically at the arithmetic involved in word problems and showed that more successful problem solvers were able to understand the deep structure rather than focusing on the context of the problem. ${ }^{9}$

\section{THIS STUDY}

This study was designed to take a slightly different approach from the above works. The goal was to look broadly at how students perceive the interaction of 
mathematics and physics and in what ways they choose which mathematical 'tools' to use during the process of problem solving. To do this, this study begins by examining the key words and phrases that students use when they are interpreting, comparing, and contrasting problems in mathematics and physics.

The portion of the study began with a problempairing task similar to those used by Rebello et al. in order to prime students to think about the interaction of mathematics and physics. As expected, the results of the pairing study showed that students did at times have difficulty thinking about the meaning of integrals, but they otherwise could approach the task logically. ${ }^{10}$ Taking into account the results by Bassok and Holyoak, a set of group interviews was conducted to determine how students paired these problems, and they were purposefully shown the mathematics problem sets first in an attempt to prime them to think about the mathematics involved in problem solving. The results of that study indicated that students may be focusing using a different set of words and symbols as clues for problem solving and what may be involved in the solution. ${ }^{11}$

\section{Methodology}

Because the process of understanding how mathematics and physics are used together is a studentcentric idea, the study seemed to lend itself toward a phenomenological approach. ${ }^{12}$ Group interviews were conducted during the 2012-2013 academic year at Mercyhurst University. Students self-selected into 8 groups of 4 based on their availability; the interviews lasted anywhere from 20-40 minutes depending on how fast the students perceived they had completed the task. All of the participants were enrolled in the second semester of a calculus-based introductory physics course.

The protocol for this phase of the study was similar to the previous phase in that it used the same set of mathematics and physics problems for continuity.
BLE 1 Sample Math and Physics problem

\begin{tabular}{|c|l|}
\hline Example Math Problem & \multicolumn{1}{|c|}{ Sample Physics Problem } \\
\hline & $\begin{array}{l}\text { A time-varying force of } \\
F(t)=3\left(t^{5}-5 t+1\right) N\end{array}$ \\
Evaluate the integral & $\begin{array}{l}\text { pushes a block along a } \\
\text { horizontal surface. How } \\
\text { much work does the force } \\
\text { exert on the block in the first } \\
\text { 8m of the motion? }\end{array}$ \\
\hline
\end{tabular}

However, the focus of this interview was different in that it focused less on the students' ideas of similarities and differences between problems and more on the ways in which they talked about interpreting math and physics problems. The overarching theme, while unsurprising, is that students tend to focus on the structure of a mathematics problem while focusing on the content of a physics problem.

In order to extend and build upon those findings, this phase of the study was meant to focus on how students identify key words and symbols while interpreting the different problems as well as the key words, phrases, and symbols they used while completing the task. As an example, Table 1 shows one math and one physics problem. A full list of the problems can be found in a previously published paper.

Audio recordings of the group interviews along with field notes from the interviewer were analyzed qualitatively according to the principles of phenomenology to extract the themes presented below.

\section{FINDINGS}

As students worked through the process of analyzing and comparing the different problems, they spoke with their group members about the ways in which the problems could be classified. For this small portion of the overall analysis, the words that students used as factors in their categorization were cataloged. It should be noted that when the students were reading the original problems aloud, those words were not counted - only those used during the actually categorization and comparison discussion.

TA

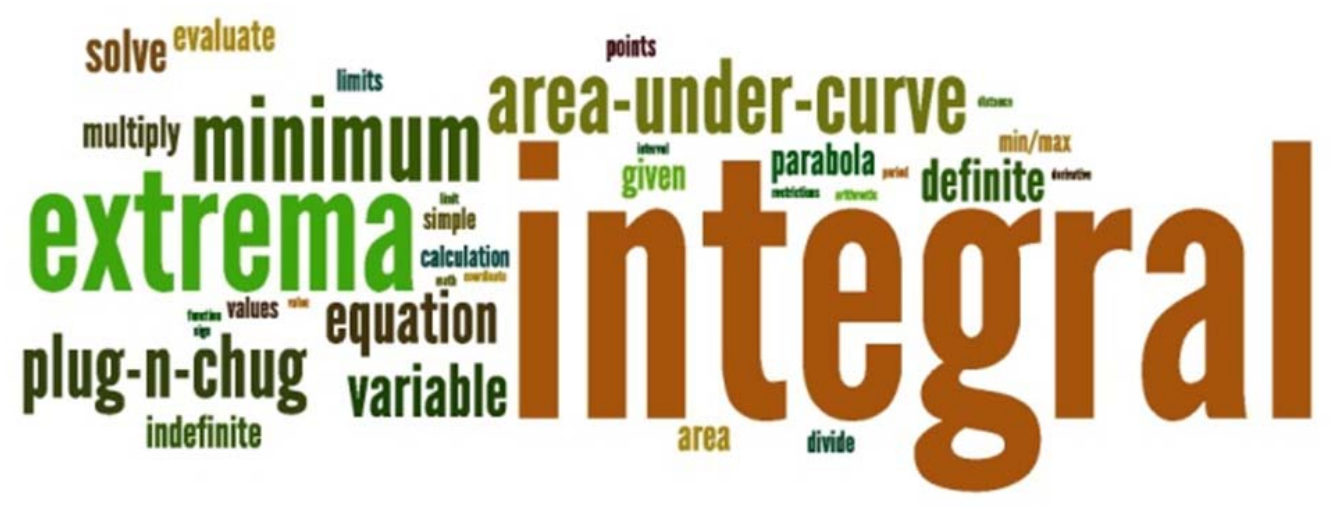

FIGURE 1. Word Cloud Diagram for the mathematics only problems. 


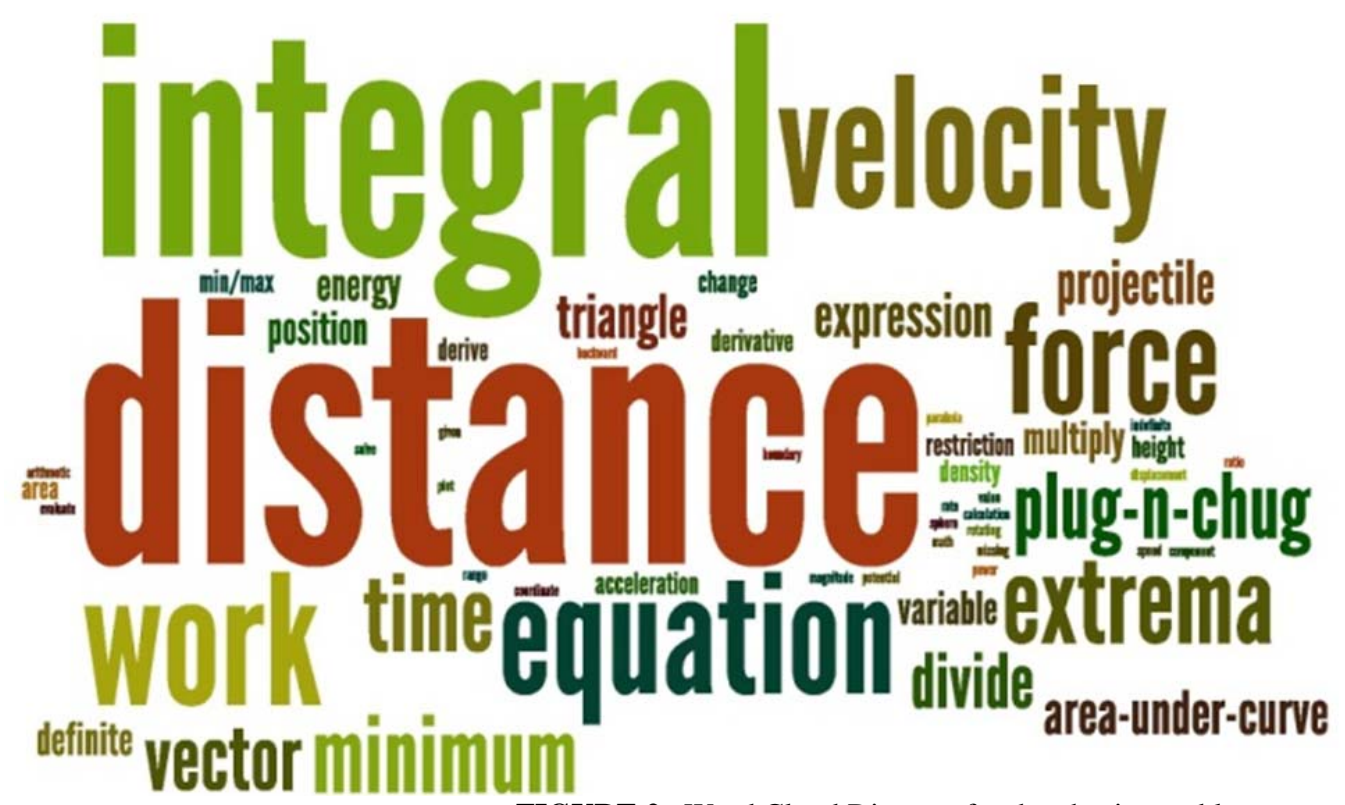

FIGURE 2. Word Cloud Diagram for the physics problems.

Figures 1 and 2 show Word Cloud Diagrams for the Mathematics and Physics problems, respectively. Word Cloud Diagrams are a visual way to indicate frequency - the larger the word, the more often it was used. Also, the scale of the diagrams is the same, so the math and physics word clouds can be compared. Table 2 contains some of the numeric data used to create the Word Cloud diagrams. Listed first is the number of distinct words used in both the math and the physics - it can be immediately seen that students use more words to describe physics problems. Also shown are the 5 most frequently used words in each type of problem, along with their frequencies.

As can be seen in Figure 1, when discussing the mathematics problems students tend to focus on what the problem is asking them to 'do'. They frequently say things like "take an integral" and every group was able to associate the idea of integration with the geometric interpretation of area-under-a-curve. It should be noted that though 4 of 10 problems had something to do with integration, that concept was discussed disproportionately with respect to the other problem types (for example, a vector addition problem as well as one that required geometry and right triangles). As previously seen within this study and other work, students often demonstrated some confusion about the meaning of definite and indefinite integrals. In fact, many students referred to definite integrals as those that had "restrictions". Such issues with vocabulary and representation may contribute to students' misconceptions about the physical meaning of different types of integration.

During the analogous task using the physics questions, a greater variety of key words and phrases was used by all of the groups, as shown in Figure 2. This task took students nearly three times as long to complete, in part because the problems were longer.
However, it is also because there was less immediate consensus about the problems. Students spent a great deal more time interpreting the problem and they also spoke in greater detail about what variables were being given and requested. Still, as shown in the Word

Cloud Diagram, students did also consider the mathematical processes that were necessary to solve the problem (though to a lesser degree than they did with the math problems).

TABLE 2. Sample frequencies of words used while interpreting math and physics problems

\begin{tabular}{|c|c|c|c|c|}
\hline & \multicolumn{2}{|l|}{ Math } & \multicolumn{2}{|c|}{ Physics } \\
\hline $\begin{array}{l}\text { \# of distinct } \\
\text { words: }\end{array}$ & \multicolumn{2}{|l|}{32} & \multicolumn{2}{|c|}{55} \\
\hline \multirow{5}{*}{$\begin{array}{l}\text { Top } 5 \\
\text { words with } \\
\text { frequencies }\end{array}$} & integral & 26 & distance & 24 \\
\hline & extrema & 12 & integral & 21 \\
\hline & minimum & 8 & velocity & 12 \\
\hline & $\begin{array}{l}\text { area under } \\
\text { curve }\end{array}$ & 7 & equation & 11 \\
\hline & plug-n-chug & 6 & work & 11 \\
\hline
\end{tabular}

The fact that "distance" was the most frequently used key word is rather intriguing, but we are unable to explain that at this time. While some of the problems did directly give or ask for a distance, it was certainly not true of all of the problems. Other "physics" terms frequently used were velocity, work, and force. "Equation" was another frequently used term, nearly always within the context of students saying that the textbook equation you would need to solve one problem may be similar to another. Though it is beyond the scope of the current project, a discourse analysis would allow for more rigorous interpretation of students' original statements. 
Also extremely evident from the Word Cloud Diagram is that students were able to focus in on the use of integrals in a number of the physics problems. This result may be surprising in some respects given the documented difficulties usually exhibited by students when it comes to the use of integration. Previous research has shown, though, that students may have more difficulty in setting up and carrying out the integral than they do in identifying the need for integration. ${ }^{5}$ However, the fact that the students were purposefully primed to focus on the mathematics is a likely explanation for this frequency. The confusion of definite and indefinite integrals was more noticeable in the physics context. For example, when students were provided with a distance interval that should have been used as the limits for an integration of force in order to find work, they considered these to be a range, a set of coordinates, or as two separate parts of the problem being asked.

\section{CONCLUSIONS}

The work presented here is a small subset of a larger study designed to examine how students view the interaction of mathematics and physics problem solving and in what ways they use the 'tools' of mathematics while solving physics problems.

It should be noted that a discussion of whether students ultimately arrived at logical comparisons is not the purpose of this portion of the study, though it will be taken into consideration in the larger analysis and future work.

This goal of this subset of the study was to look at the key words and phrases students use to interpret, compare, and contrast problems in both physics and mathematics. The results suggest that students tend to focus on the context of the physics problems to a great degree, even when they are able to also discuss the mathematical tools necessary to complete the solution. There are many potential explanations for this phenomenon, all of which could be explored in future research. It may be the classic expert/novice dilemma as illustrated by Chi and colleagues - the novice students are distracted by the surface features and therefore do not focus on the underlying mathematical tools and structures. However, it may also be that the problems were unwittingly designed to have more 'interesting' information than necessary, which has been shown to be detrimental to student learning. ${ }^{13}$

Perhaps most significantly for the future of this work, these results provide a phenomenological basis for the way that students approach these problems and will enable a research-based intervention to be designed that may help students think about the mathematics that will be helpful during their solution path. In particular, it may be helpful to study what interventions help students to focus on the mathematical tools and solution paths and not on the content or surface features of the problem. The next step toward that end could be to move forward with this study and have the students actually carry out a solution to the problems. By doing so, we may be able to determine whether the key words and phrases they use is related in some way to the process of solving the problems - both in terms of approach and correctness.

\section{ACKNOWLEDGEMENTS}

This research was supported by an in-house Faculty Research Fellowship position at Mercyhurst University.

\section{REFERENCES}

1. M. Bassok, and K. J. Holyoak, Journal of Experimental Psychology 15, 153 (1989).

2. N. S. Rebello et al., in Learning to Solve Complex Scientific Problems, edited by D. H. Jonassen (Lawrence Earlbaum, New York, 2007).

3. E. F. Redish, in World View on Physics Education in 2005: Focusing on ChangeDelhi, 2005).

4. J. F. Wagner, C. Manogue, and J. R. Thompson, AIP Conference Proceedings 1413, 89 (2011).

5. R. R. Bajracharya, T. R. Wemyss, and J. R. Thompson, AIP Conference Proceedings 1413, 111 (2011).

6. D. Hu, J. Von Korff, and N. S. Rebello, AIP Conference Proceedings 1413, 215 (2011).

7. N. Khan et al., AIP Conference Proceedings 1413, 227 (2011).

8. D.H. Nguyen, and N. S. Rebello, Physical Review Special Topics PER 7 (2011).

9. D.H. Nguyen, and N. S. Rebello, Physical Review Special Topics PER 7 (2011).

10. M. T. H. Chi, P. J. Feltovich, and R. Glaser, Cognitive Science 52, 121 (1981).

11. P. T. Hardiman, R. J. Dufresne, and J. Mestre, Memory and Cognition 17, 627 (1989).

12. C. Singh, American Association of Physics Teachers, American Journal of Physics 77, 73 (2009).

13. M. Hegarty, R. E. Mayer, and C. A. Monk, Journal of Educational Psychology 87, 18 (1995).

14. D. L. McBride, AIP Conference Proceedings 1413, 275 (2011).

15. D. L. Jones, and R. B. Roseman, AIP Conference Proceedings 1513, 206 (2011).

16. F. Marton, Journal of Thought 21, 28 (1986).

17. R. E. Mayer et al., Journal of Experimental Psychology 14, 329 (2008). 